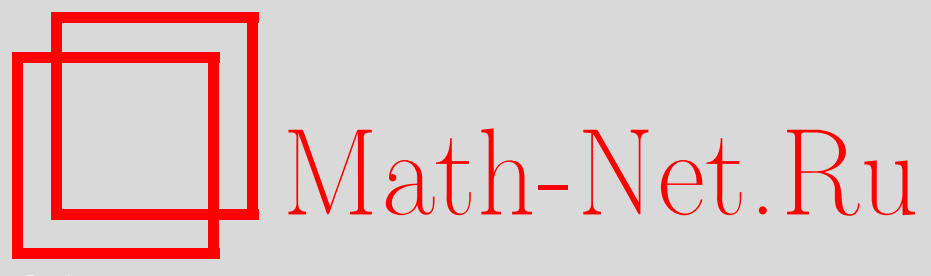

И. А. Тайманов, С. П. Царев, Двумерные операторы Шрёдингера с быстро убывающим рациональным потенциалом и многомерным $L_{2}$-ядром, $У M H, 2007$, том 62 , выпуск 3, 217-218

DOI: https://doi.org/10.4213/rm6817

Использование Общероссийского математического портала Math-Net.Ru подразумевает, что вы прочитали и согласны с пользовательским соглашением http://www.mathnet.ru/rus/agreement

Параметры загрузки:

IP: 35.173 .137 .237

26 апреля 2023 г., 15:06:27

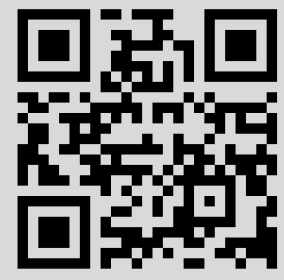




\title{
Двумерные операторы Шрёдингера с быстро убывающим рациональным потенциалом и многомерным $L_{2}$-ядром
}

\author{
И. А. Тайманов, С. П. Царёв
}

В этой заметке мы указываем, как с помощью преобразования Мутара построить явные примеры двумерных операторов Шрёдингера $L=-\Delta+u(x, y)$ с быстро убывающим потенциалом и многомерным ядром, содержащим гладкие квадратично суммируемые функции. В указанных ниже явных примерах потенциал $u(x, y)$ и квадратично суммируемые решения $\psi(x, y)$ уравнения $L \psi=0$ являются гладкими рациональными функциями от $x$ и $y$. В наилучшем указанном случае потенциал $u$ и собственные функции $\psi$ убывают как $1 / r^{8}$ и $1 / r^{3}$ соответственно, где $r^{2}=x^{2}+y^{2}$. Построенные потенциалы являются точно решаемыми на нулевом уровне энергии в том смысле, что все решения уравнения $L \psi=0$ строятся по гармоническим функциям и построение сводится к квадратурам.

Для операторов с потенциалами, убывающими настолько быстро, существует хорошая спектральная теория [1], [2]. В одномерном случае существование квадратично суммируемых собственных функций на нулевом уровне энергии невозможно. Обратная спектральная задача для многомерных операторов Шрёдингера на фиксированном уровне энергии была впервые поставлена в [3], где рассматривались двоякопериодические потенциалы. В случае быстро убывающих потенциалов она исследовалась в основном для уровней энергии, которые либо положительны [4], либо лежат ниже основного состояния [5].

Преобразование Мутара сопоставляет двумерному оператору Шрёдингера

$$
L=-\Delta+u=-\left(\frac{\partial^{2}}{\partial x^{2}}+\frac{\partial^{2}}{\partial y^{2}}\right)+u(x, y)
$$

оператор Шрёдингера $\tilde{L}$ с потенциалом $\tilde{u}: \tilde{L}=-\Delta+\tilde{u}=-\Delta+(u-2 \Delta \log \omega)$, где $\omega-$ некоторое решение уравнения $L \omega=0$, что влечет равенство $\tilde{u}=2\left(\omega_{x}^{2}+\omega_{y}^{2}\right) / \omega^{2}-u$. По каждому решению $\varphi$ уравнения $L \varphi=0$ строится решение $\tilde{\varphi}$ уравнения $\tilde{L} \tilde{\varphi}=0$ : $(\omega \tilde{\varphi})_{x}=-\omega^{2}\left(\frac{\varphi}{\omega}\right)_{y},(\omega \tilde{\varphi})_{y}=\omega^{2}\left(\frac{\varphi}{\omega}\right)_{x}$. Построение $\tilde{\varphi}$ по $\varphi$ сводится к квадратурам и функция $\tilde{\varphi}$ однозначно определена по модулю слагаемых вида $C \omega^{-1}$, где $C=$ const. Учитывая эту неоднозначность, мы для удобства обозначим преобразование Мутара через $M_{\omega}: \tilde{u}=M_{\omega}(u), \tilde{\varphi}=M_{\omega}(\varphi)$. Отметим также, что $\tilde{L} \omega^{-1}=0$.

В случае, когда потенциал $u$ зависит только от $x: u=u(x)$, и $\omega(x, y)=f(x) e^{k y}$, мы имеем $L=-\frac{\partial^{2}}{\partial y^{2}}+A^{\top} A+k^{2}$, где $A=-\frac{\partial}{\partial x}+\frac{f_{x}}{f}, A^{\top}=\frac{\partial}{\partial x}+\frac{f_{x}}{f}$, и преобразование Мутара сводится к преобразованию Дарбу одномерного оператора $L^{\prime}=-\frac{\partial^{2}}{\partial x^{2}}+\left(u+k^{2}\right)$, а именно $L \rightarrow \tilde{L}=-\frac{\partial^{2}}{\partial y^{2}}+A A^{\top}+k^{2}$. Итерации преобразования Дарбу, начинающиеся с потенциала $u_{0}=0$ и $f(x)=x$, дают все точно решаемые рациональные одномерные потенциалы [6]. Все эти потенциалы сингулярны.

Общая схема построения. Рассмотрим преобразования Мутара оператора Шрёдингера с потенциалом $u_{0}$, отвечающие функциям $\omega_{1}$ и $\omega_{2}$, и обозначим соответствующие потенциалы через $u_{1}$ и $u_{2}$. Функции вида $M_{\omega_{1}}\left(\omega_{2}\right)$ определены с точностью до слагаемых вида $C / \omega_{1}$ и удовлетворяют уравнению $\left(-\Delta+u_{1}\right) \psi=0$. Аналогичное верно для функций вида $M_{\omega_{2}}\left(\omega_{1}\right)$. Зафиксируем некоторую функцию $\theta_{1}$ из класса $M_{\omega_{1}}\left(\omega_{2}\right)$

Работа выполнена при поддержке РФФИ (гранты 06-01-00094a, 06-01-00814a), комплексного интеграционного проекта $2.15 \mathrm{CO}$ РАН, Института Макса Планка по математике (И.А.Т.) и DFG Research Unit 565 "Polyhedral Surfaces" (TU-Berlin, С.П.Ц.). 
и в качестве представителя класса $M_{\omega_{2}}\left(\omega_{1}\right)$ возьмем функцию $\theta_{2}=-\left(\omega_{1} / \omega_{2}\right) \theta_{1}$. Применим теперь к оператору $\left(-\Delta+u_{1}\right)$ преобразование Мутара, отвечающее функции $\theta_{1}$, а к оператору $\left(-\Delta+u_{2}\right)-$ преобразование Мутара, отвечающее функции $\theta_{2}$. Имеет место известная лемма.

Лемма 1. $M_{\theta_{1}}\left(u_{1}\right)=M_{\theta_{2}}\left(u_{2}\right)=u$, а функиии $\psi_{1}=1 / \theta_{1} u \psi_{2}=1 / \theta_{2}$ удовлетворяют уравнению $L \psi=0$, где $L=-\Delta+u$.

В этой конструкции есть свободный параметр, связанный с выбором функции $\theta_{1}$ из класса $M_{\omega_{1}}\left(\omega_{2}\right)$. Он в ряде случаев позволяет контролировать несингулярность потенциала $u$ и функций $\psi_{1}$ и $\psi_{2}$.

ПримеР 1. Пусть $u_{0}=0, \omega_{1}=x+2\left(x^{2}-y^{2}\right)+x y$ и $\omega_{2}=x+y+\frac{3}{2}\left(x^{2}-y^{2}\right)+5 x y$. При некотором выборе $\theta_{1}$ (мы опускаем явную формулу вследствие ее громоздкости) мы получаем

$$
\begin{gathered}
u=-\frac{5120\left(1+8 x+2 y+17 x^{2}+17 y^{2}\right)}{\left(160+4 x^{2}+4 y^{2}+16 x^{3}+4 x^{2} y+16 x y^{2}+4 y^{3}+17\left(x^{2}+y^{2}\right)^{2}\right)^{2}}, \\
\psi_{1}=\frac{x+2 x^{2}+x y-2 y^{2}}{160+4 x^{2}+4 y^{2}+16 x^{3}+4 x^{2} y+16 x y^{2}+4 y^{3}+17\left(x^{2}+y^{2}\right)^{2}}, \\
\psi_{2}=\frac{2 x+2 y+3 x^{2}+10 x y-3 y^{2}}{160+4 x^{2}+4 y^{2}+16 x^{3}+4 x^{2} y+16 x y^{2}+4 y^{3}+17\left(x^{2}+y^{2}\right)^{2}} .
\end{gathered}
$$

Теорема 1. Потенциал и вида (1) является гладким, рациональным и убывает $\kappa a \kappa 1 / r^{6}$ npu $r \rightarrow \infty$.

Функции $\psi_{1}$ и $\psi_{2}$ вида (2) - гладкие, рациональные, убывают как $1 / r^{2}$ при $r \rightarrow \infty$ u порождают двумерное подпространство в ядре оператора $L=-\Delta+u: L_{2}\left(\mathbb{R}^{2}\right) \rightarrow$ $L_{2}\left(\mathbb{R}^{2}\right)$.

ПримеР 2. Пусть $u_{0}=0, \omega_{1}=x+\frac{x^{2}-y^{2}-3 x y}{5}+2\left(-x^{3}-3 x^{2} y+3 x y^{2}+y^{3}\right)$ и $\omega_{2}=x+y+\frac{x^{2}-y^{2}}{2}-\frac{x y}{5}-4\left(3 x^{2} y-y^{3}\right)$. При хорошем выборе $\theta_{1}$ мы получаем гладкий рациональный потенциал $u$, убывающий как $1 / r^{8}$, и гладкие рациональные функции $\psi_{1}$ и $\psi_{2}$, лежащие в $L_{2}$-ядре оператора Шрёдингера с потенциалом $u$ и убывающие как $1 / r^{3}$. Мы опускаем явные формулы вследствие их громоздкости.

По-видимому, увеличивая степень затравочных гармонических полиномов $\omega_{1}$ и $\omega_{2}$, мы для любого $N>0$ можем получить потенциалы $u$ и собственные функции $\psi_{1}$ и $\psi_{2}$, убывающие быстрее, чем $1 / r^{N}$.

Авторы благодарят П. Г. Гриневича и С. П. Новикова за полезные обсуждения.

\section{Список литературы}

[1] Л. Д. Фаддеев, “Обратная задача квантовой теории рассеяния”, Итоги науки и техники. Соврем. проблемы матем., 3, ВИНИТИ, М., 1974, 93-180. [2] Р. Г. Новиков, Г. М. Хенкин, УМH, 42:3 (1987), 93-152. [3] Б. А. Дубровин, И. М. Кричевер, С. П. Новиков, Докл. АН СССР, 229:1 (1976), 15-19.[4] П. Г. Гриневич, С. В. Манаков, Функи. анализ и его прилож., 20:2 (1986), 14-24. [5] П. Г. Гриневич, С. П. Новиков, Функи. анализ и его прилож., 22:1 (1988), 23-33. [6] H. Airault, H. P. McKean, J. Moser, Comm. Pure Appl. Math., 30:1 (1977), $95-148$.

\section{И. А. Тайманов (I. A. Taimanov)}

Институт математики СО РАН, Новосибирск

E-mail: taimanov@math.nsc.ru
Представлено С. П. Новиковым Принято редколлегией 20.04.2007

\section{C. П. Царёв (S. P. Tsarev)}

Institut für Mathematik, Technische Universität Berlin, Germany;

Красноярский государственный педагогический университет

E-mail: sptsarev@mail.ru 